\title{
DUALITY FOR HADAMARD PRODUCTS WITH APPLICATIONS TO EXTREMAL PROBLEMS FOR FUNCTIONS REGULAR IN THE UNIT DISC
}

\author{
BY

\section{STEPHAN RUSCHEWEYH}

ABSTRACT. Let $A$ be the set of functions regular in the unit disc $U$ and $A_{0}$ the set of all functions $f \in A$ which satisfy $f(0)=1$. For $V \subset A_{0}$ define the dual set $V^{*}=\left\{f \in A_{0} \mid f * g \neq 0\right.$ for all $\left.g \in V, z \in U\right\}, V^{* *}=\left(V^{*}\right)^{*}$. Here $f * g$ denotes the Hadamard product. THEOREM. Let $V \subset A_{0}$ have the following properties: (i) $V$ is compact, (ii) $f \in V$ implies $f(x z) \in V$ for all $|x| \leqslant 1$. Then $\lambda(V)=\lambda\left(V^{* *}\right)$ for all continuous linear functionals $\lambda$ on $A$. This theorem has many applications to functions in $A$ which are defined by properties like bounded real part, close-to-convexity, univalence etc.

Introduction. Let $f(z)=\Sigma_{k=0}^{\infty} a_{k} z^{k}$ be regular for $|z|<R_{1}, g(z)=$ $\Sigma_{k=0}^{\infty} b_{k} z^{k}$ regular for $|z|<R_{2}$. The Hadamard product of $f$ and $g$ is defined by $(f * g)(z)=\Sigma_{k=0}^{\infty} a_{k} b_{k} z^{k}$. This new function is regular for $|z|<R_{1} R_{2}$. Let $U$ be the unit disc $\{|z|<1\}, \bar{U}$ its closure. By $A, \bar{A}$ we denote the set of functions regular in $U$ and $\bar{U}$ respectively. Furthermore let $A_{0}$ be the subset of $A$ whose elements $f(z)$ are normalized by $f(0)=1$.

In this paper we introduce a certain duality between subsets of $A_{0}$ by means of the Hadamard product:

Definition. For $V \subset A_{0}$, we call the set $V^{*}=\left\{g \in A_{0} \mid f * g \neq 0\right.$ for all $f \in V, z \in U$ \} dual to $V$.

We are interested in relations between $V$ and $V^{* *}=\left(V^{*}\right)^{*}$. In general, $V^{* *}$ is much greater than $V$, but a lot of properties of $V$ remain valid in $V^{* *}$. Our main result, although its proof is simple, has many applications to classes of functions which are defined by properties like bounded real part, convexity, starlikeness, close-to-convexity, univalence etc. A special case of our method was already treated in [10]. The applications that we give in the second section of this paper are numerous, and some new and perhaps interesting information is given. Nevertheless they are merely thought to be examples illustrating the method, and much work remains to be done.

Received by the editors March 6, 1974 and, in revised form, June 3, 1974.

AMS (MOS) subject classifications (1970). Primary 30A40, 30A32.

Key words and phrases. Hadamard product, continuous linear functionals, functions with bounded real part, close-to-complex functions, partial sums, Marx conjecture. 
I. The main theorem. Obviously we have $V \subset V^{* *}$ for every $V \subset A_{0}$ and it is easily seen that equality holds if and only if $V$ is a dual set, i.e. there exists a $W \subset A_{0}$ with $V=W^{*}$. It would be very interesting to find intrinsic conditions under which $V$ is a dual set but here we are not concerned with this problem. We shall consider a similar but somewhat weaker question, more flexible in the applications.

Let $\Lambda$ be the set of all continuous (w.r.t. compact convergence in $U$ ) linear functionals on $A$. Our problem is to find conditions for $V \subset A_{0}$ such that $\lambda(V)=\lambda\left(V^{* *}\right)$ holds for all $\lambda \in \Lambda$. We shall see that the combination of the following not very strong conditions already forces this property:

(i) $V$ is compact.

(ii) If $f \in V$, then so is $f_{x}(z)=f(x z),|x| \leqslant 1$.

In fact, it is possible to replace (ii) by the following weaker condition (ii') which looks somewhat artificial but is useful on several occasions.

(ii') Let $V^{\prime}=\left\{g \in A_{0} \mid \exists(f \in V,|x| \leqslant 1): g(z)=f_{x}(z)\right\}$. Then $0 \notin$ $\lambda(V)$ implies $0 \notin \lambda\left(V^{\prime}\right)$ for all $\lambda \in \Lambda$.

Clearly (ii) implies (ii') since $V^{\prime}=V$ in this case.

REMARK 1. Every dual set fulfils (ii).

MAIN THEOREM. Let $V \subset A_{0}$ fulfil (i), (ii'). Then $f \in A_{0}$ is a member of $V^{* *}$ if and only if $\lambda(f) \in \lambda(V)$ for all $\lambda \in \Lambda$. In particular we have $\lambda(V)=$ $\lambda\left(V^{* *}\right)$ for all $\lambda \in \Lambda$.

An important tool in the proof will be the following lemma due to 0 . Toeplitz [12].

LEMma 1. $\lambda \in \Lambda$ if and only if there exists a function $g \in \bar{A}$ such that $\lambda(f)=(g * f)(1)$ for all $f \in A$.

In the sequel we shall denote this correspondence by $\lambda \doteqdot g$.

Proof of the MAIN THEOREM. We start with the proof of the second assertion. Let

$$
\widetilde{V^{*}}=\left\{f \in V^{*} \mid \exists\left(r \in(0,1), h \in V^{*}\right): f(z)=h(r z)\right\} .
$$

For $\lambda \in \Lambda, \lambda \doteqdot g$, we define

$$
X_{\lambda}=\left\{w \mid \frac{g(z)-w}{g(0)-w} \notin \widetilde{V^{*}}\right\}
$$

and remark that $g(0) \in X_{\lambda}$. Now we need several steps.

(1) Let $w \notin X_{\lambda}$, i.e. $(g(z)-w) /(g(0)-w) \in \widetilde{V^{*}}$. Let $h(r z)=$ $(g(z)-w) /(g(0)-w)$, where $h \in V^{*}, r \in(0,1)$. For all $f \in V^{* *}$ we have 


$$
\begin{aligned}
0 & \neq(h(z) * f(z))(r)=(h(r z) * f(z))(1) \\
& =\frac{1}{g(0)-w}[(g(z)-w) * f(z)](1)=\frac{1}{g(0)-w}(\lambda(f)-w),
\end{aligned}
$$

and consequently $w \notin \lambda\left(V^{* *}\right)$. This implies $\lambda\left(V^{* *}\right) \subset X_{\lambda}$.

(2) Let $\mu \in \Lambda, \mu \doteqdot p$, and, for small $|\sigma-1|$, put $\mu_{\sigma} \doteqdot p(\sigma z)$. If $0 \notin$ $\mu(V)$, it follows from the compactness of $V$ that there exists a $\sigma>1$ such that $0 \notin \mu_{\sigma}(V)$.

(3) We prove $g(0) \in \lambda(V)$. Assuming the contrary we have $0 \notin \lambda^{\prime}(V)$ for $\lambda^{\prime} \doteqdot g(z)-g(0)$, and from (ii') it follows $0 \notin \lambda^{\prime}\left(V^{\prime}\right)$. But $f_{0}(z) \equiv 1 \in V^{\prime}$ and $\lambda^{\prime}\left(f_{0}\right)=\left[(g(z)-g(0)) * f_{0}(z)\right](1)=0$.

(4) Now let $w \notin \lambda(V)$, so that $[(g(z)-w) * f(z)](1) \neq 0, f \in V$.

From (2) we have the existence of $\sigma>1$ with $[(g(z)-w) * f(z)](\sigma) \neq 0, f \in V$, and (ii') implies $[(g(z)-w) * f(z)](x) \neq 0,|x| \leqslant \sigma$. Therefore we can conclude $(g(z)-w) /(g(0)-w) \in \widetilde{V^{*}}$ which gives $X_{\lambda} \subset \lambda(V)$. From the obvious relation $\lambda(V) \subset \lambda\left(V^{* *}\right)$ and (1) the result follows.

It remains to prove, that a function $f \in A_{0}$ which fulfils $\lambda(f) \in \lambda(V)$ for all $\lambda \in \Lambda$ is a member of $V^{* *}$. In fact, for every $\lambda \doteqdot g \in \widetilde{V^{*}}$ we have $\lambda(f)=$ $(g * f)(1) \neq 0$, since $0 \notin \lambda(V)$. If $h \in V^{*}$, then $h_{x}(z)=h(x z) \in \widetilde{V}^{*}$ for every $|x|<1$ (Remark 1). Consequently $(h * f)(x)=\left(h_{x} * f\right)(1) \neq 0$ and $f \in V^{* *}$ follows.

Next we mention three corollaries of our theorem that increase its applicability.

Corollary 1. Let $V \subset A_{0}$ fulfil (i), (ii'). Let $\lambda_{1}, \lambda_{2} \in \Lambda, 0 \notin \lambda_{2}(V)$. Then, for every $f \in V^{* *}$, there exists a $g \in V$ such that $\lambda_{1}(f) / \lambda_{2}(f)=$ $\lambda_{1}(g) / \lambda_{2}(g)$. includes

Proof. Let $\lambda_{(u)}=\lambda_{1}+u \lambda_{2}, u \in \mathrm{C}$. Then $\lambda_{(u)}(V)=\lambda_{(u)}\left(V^{* *}\right)$, which $\lambda_{1}(f) / \lambda_{2}(f) \neq u$ for all $f \in V \Longleftrightarrow \lambda_{1}(f) / \lambda_{2}(f) \neq u$ for all $f \in V^{* *}$.

The result follows immediately.

Corollary 2. Let $V \subset A_{0}$ fulfil (i), (ii'). Then the closed convex hulls of $V$ and $V^{* *}$ are equal.

PROOF. This is a consequence of the main theorem and of a general separation theorem in locally convex linear topological spaces [5, p. 119].

The following observation leads to Corollary 3. 
Lemma 2. Let $V, W \subset A_{0}, g \in A_{0}$. Let

$$
\begin{aligned}
& r_{1}=\sup \left\{r \mid(g * f)(r z) \in W^{*} \text { for all } f \in V^{* *}\right\}, \\
& r_{2}=\sup \left\{r \mid(g * f)(r z) \in V^{*} \text { for all } f \in W^{* *}\right\} .
\end{aligned}
$$

Then $\min \left(r_{1}, 1\right)=\min \left(r_{2}, 1\right)$.

Proof. Let $r \leqslant \min \left(r_{1}, 1\right)$. If $(g * f)(r z) \in W^{*}$ for all $f \in V^{* *}$, we have $(g * f * h)(r z) \neq 0, z \in U$, for all $h \in W^{* *}$. This implies $(g * h)(r z) \in\left(V^{* *}\right)^{*}$ $=V^{*}$ for all $h \in W^{* *}$ and so $r \leqslant r_{2}$. After interchanging $V$ and $W$ the result follows.

Corollary 3. Let $V, W \subset A_{0}, g \in A_{0}$, and let $V$ fulfil (i), (ii'). Then we have $\min \left(r_{1}, 1\right)=\min \left(r_{1}^{\prime}, 1\right)$, where $r_{1}$ is the number defined in Lemma 2 and $r_{1}^{\prime}=\sup \left\{r \mid(g * f)(r z) \in W^{*}\right.$ for all $\left.f \in V\right\}$.

Proof. Clearly $\min \left(r_{1}^{\prime}, 1\right) \geqslant \min \left(r_{1}, 1\right)$. Let $\min \left(r_{1}^{\prime}, 1\right)>r>\min \left(r_{1}, 1\right)$, $h \in W$, and $\lambda \doteqdot(h * g)(r z)$. In this case $(f * g)(r z) \in \widetilde{W^{*}}$ for every $f \in V$, so that we have $\lambda(f)=(h(z) * g(r z) * f(z))(1) \neq 0$, and, by the main theorem, the same is true for all $f \in V^{* *}$. Since $V^{* *}$ fulfils (ii), we can conclude $(h(z) * g(r z) * f(z))(z) \neq 0, z \in U$, and this means, by definition, $(g * f)(r z) \in$ $W^{*}$ for all $f \in V^{* *}$. So $r \leqslant r_{1}$, in contradiction to the assumption.

\section{Applications.}

1. The first problem in using the main theorem is to construct the set $V^{* *}$ for a given $V \subset A_{0}$, or, in turn, to find for a given interesting set $V^{* *}$ a set $V$ which is small enough to provide real gain. The following sets correspond to widely used subsets of $A_{0}$.

$$
\begin{aligned}
& V_{\beta}=\left\{(1-\beta) \frac{1-x z}{1-y z}+\beta|| x|=| y \mid=1\right\}, \quad \beta \in \mathbf{R}, \beta \neq 1, \\
& V_{\beta 0}=\left\{\gamma(1-\beta) \frac{1+x z}{1-x z}+(1-\gamma)(1-\beta) \frac{1+y z}{1-y z}+\beta \mid\right. \\
& \qquad|x|=|y|=1,0 \leqslant \gamma \leqslant 1\}, \beta \in \mathbf{R}, \beta \neq 1, \\
& \hat{X}=\left\{\frac{1+1 / 2(y-x) z}{(1-x z)^{2}} \mid(|x|=|y|=1) \wedge(x=y,|x|<1)\right\}, \\
& \hat{Y}=\left\{\frac{1}{(1-x z)(1-y z)}|| x|=| y \mid=1\right\} .
\end{aligned}
$$

Obviously all of these sets are compact and we only have to check (ii'). Let $\lambda \in \Lambda, \lambda \doteqdot g$. It is clear, that it is enough to assume (a) $g(0)=0$ or (b) $g(0)=1$. 


$$
\begin{aligned}
V_{\beta}:(g *[(1-\beta) & \left.\left.\frac{1-x z}{1-y z}+\beta\right]\right)(1) \\
& =(1-\beta)[x / y+(1-x / y) g(y)]+\beta g(0),
\end{aligned}
$$

and $0 \notin \lambda\left(V_{\beta}\right)$ implies

(a) $g(y) \neq 1 / 2+i \eta$,

(b) $g(y) \neq(1-2 \beta) /(2-2 \beta)+i \eta$,

for $|y|=1, \eta \in \mathbf{R}$. In both cases $g(y),|y|=1$, lies in a certain halfplane and, of course, the inequalities (a), (b) remain valid for $(|x|=)|y|<1$. (ii') follows.

$$
\begin{aligned}
V_{\beta 0}:(g *[\gamma(1-\beta) & \left.\left.\frac{1+x z}{1-x z}+(1-\gamma)(1-\beta) \frac{1+y z}{1-y z}+\beta\right]\right)(1) \\
& =(1-\beta)[\gamma(2 g(x)-1)+(1-\gamma)(2 g(y)-1)]+\beta g(0) .
\end{aligned}
$$

$0 \notin \lambda\left(V_{\beta 0}\right)$ implies

(a) $\gamma(g(x)-1 / 2)+(1-\gamma)(g(y)-1 / 2) \neq 0$,

(b) $\gamma(g(x)-(1-2 \beta) /(2-2 \beta))+(1-\gamma)(g(y)-(1-2 \beta) /(2-2 \beta)) \neq 0$, for $|x|=|y|=1,0 \leqslant \gamma \leqslant 1$. As before it is not difficult to see, that (a), (b) remain valid for $|x|=|y|<1$, and again (ii') follows.

$\hat{X}$ : This case was already treated in [10], but for the sake of completeness we give a sketch of the proof:

$$
\left(g * \frac{1+1 / 2(y-x) z}{(1-x z)^{2}}\right)(1)=1 / 2(1-y / x) g(x)+1 / 2(1+y / x)(x g(x))^{\prime},
$$

and $0 \notin \lambda(\hat{X})$ implies firstly $(x g(x))^{\prime} \neq 0,|x| \leqslant 1$, and secondly

$$
\frac{g(x)}{(x g(x))^{\prime}} \neq \frac{y+x}{y-x}, \quad|x|=|y|=1 .
$$

This gives, by the minimum principle for harmonic functions, the desired result.

$$
\hat{Y}:\left(g * \frac{1}{(1-x z)(1-y z)}\right)(1)=\frac{x g(x)-y g(y)}{x-y}
$$

and $0 \notin \lambda(\hat{Y})$ implies the global univalence of $x g(x)$ in $|x| \leqslant 1$. (ii') follows.

We now introduce the following notations:

$$
\begin{aligned}
P_{\beta} & =\left\{f \in A_{0} \mid \exists \alpha \in \mathbf{R}: \operatorname{Re} e^{i \alpha}(g(z)-\beta)>0, z \in U\right\}, \\
P_{\beta 0} & =\left\{f \in A_{0} \mid \operatorname{Re} \frac{g(z)-\beta}{1-\beta}>0, z \in U\right\}, \\
\widehat{S t} & =\left\{f \in A_{0} \mid z f(z) \text { univalent and starlike in } U\right\},
\end{aligned}
$$




$$
\hat{S}=\left\{f \in A_{0} \mid z f(z) \text { univalent in } U\right\} .
$$

Furthermore we put $\hat{Z}=(\hat{S})^{*}, \hat{M}=(\widehat{S t})^{*}$.

THEOREM 1. $V_{\beta}^{* *}=P_{\beta}, V_{\beta 0}^{* *}=P_{\beta 0}, \hat{X}^{* *}=\hat{M}, \hat{Y}^{* *}=\hat{Z}$.

Proof. From our considerations to prove (ii') for these sets, it is easily seen, that

$$
V_{\beta}^{*}=P_{\delta 0}, \quad V_{\beta 0}^{*}=P_{\delta}, \quad \hat{X}^{*}=\widehat{S t}, \quad \hat{Y}^{*}=\hat{S},
$$

holds. Here $\delta=(1-2 \beta) /(2-2 \beta)$. The set $P_{\delta 0}$ consists of the functions

$$
g(z)=\int_{0}^{2 \pi}\left[(1-\delta) \frac{1+z e^{-i \varphi}}{1-z e^{-i \varphi}}+\delta\right] d \mu(\varphi)
$$

where $\mu(\varphi)$ denotes an arbitrary probability mass on $[0,2 \pi]$. For $f \in A_{0}$ we have

$$
(f * g)(z)=\int_{0}^{2 \pi}\left[(1-\delta)\left(2 f\left(z e^{-i \varphi}\right)-1\right)+\delta\right] d \mu(\varphi),
$$

and if, for fixed $z, g$ traverses $P_{\delta 0}$, then $(f * g)(z)$ traverses the closed convex hull of $\left\{(1-\delta)\left(2 f\left(z e^{-i \varphi}\right)-1\right)+\delta \mid 0 \leqslant \varphi \leqslant 2 \pi\right\}$. So $f \in V_{\beta}^{* *}$ implies the existence of $\alpha \in \mathbf{R}$, such that

$$
\operatorname{Re} e^{i \alpha}(f(z)-(1-2 \delta) /(2-2 \delta))>0, \quad z \in U,
$$

holds, which gives $f \in P_{\beta}$. The other direction follows similarly.

In the case $V_{\beta 0}$ we only have to observe the relation $V_{\beta 0}^{* *}=P_{\delta}^{*}=P_{\beta 0}^{* *}$. But $P_{\beta 0}$ is a dual set $\left(V_{\delta}^{*}=P_{\beta 0}\right)$ and so $P_{\beta 0}^{* *}=P_{\beta 0}$. The other two statements of the theorem follow immediately from (1) and the definition of $\hat{M}, \hat{Z}$.

2. Applications to $P_{\beta}$. From the great variety of possible applications of the main theorem in the cases $P_{\beta}$ (and $P_{\beta 0}$ ) we mention only two typical ones. The $n$th partial sum $\Sigma_{k=0}^{n} a_{k} z^{k}$ of a function $f(z)=\Sigma_{k=0}^{\infty} a_{k} z^{k}$ is denoted by $\pi_{n}(z, f)$.

Theorem 2. Let $g \in P_{\beta}, 0 \leqslant \beta<1$. Then, for $n=0,1, \ldots$, we have the sharp inequality

$$
\left|\pi_{n}(z, g) / g(z)-1\right| \leqslant \begin{cases}\sqrt{1 / \beta-1} \frac{|z|^{n+1}}{\sqrt{1-|z|^{2}}}, & 0<\beta<1, \\ \frac{2|z|^{n+1}}{1-|z|}, & \beta=0 .\end{cases}
$$

Proof. For fixed $z \in U$ the expression $\pi_{n}(z, g) / g(z)$ is the quotient of two continuous linear functionals, and we have $g(z) \neq 0$. Therefore we can apply 
Corollary 1 to $V_{\beta}$ and obtain

$$
\begin{aligned}
\left|\frac{\pi_{n}(z, g)}{g(z)} \quad 1\right| & =\left|\frac{(1-\beta) y^{n}(x-y) z^{n+1}}{1-[(1-\beta) x+\beta y] z}\right| \\
& \leqslant(1-\beta)|z|^{n+1} \max _{|w|=1} \frac{|1-w|}{1-|1-\beta(1-w)||z|} .
\end{aligned}
$$

The simple evaluation of the maximum gives the result.

T. H. MacGregor [6] proved that every function $z g(z)$, where $g \in P_{00}$, is starlike and univalent for $|z|<\sqrt{2}-1$. It is not difficult to see, that his result is also true for $z g(z), g \in P_{0}$. Our next theorem is concerned with the radius of convexity of these functions.

THEOREM 3. Let $g \in P_{0}$. Then, for $f(z)=z g(z)$, we have the sharp inequality

$$
\operatorname{Re}\left[\frac{z f^{\prime \prime}(z)}{f^{\prime}(z)}+1\right] \geqslant \frac{1-5|z|-3|z|^{2}-|z|^{3}}{(1+|z|)\left(1-2|z|-|z|^{2}\right)}, \quad|z|<\sqrt{2}-1 .
$$

This implies, that $f(z)$ is convex for

$$
|z|<(\sqrt{116 / 27}+2)^{1 / 3}-(\sqrt{116 / 27}-2)^{1 / 3}-1=0.179 \cdots .
$$

Proof. It is enough to evaluate this functional for $f_{0}(z)=$ $z(1+x z) /(1+y z),|x|=|y|=1$. We have

$$
\frac{z f_{0}^{\prime \prime}(z)}{f_{0}^{\prime}(z)}+1=\frac{2 x\left(z+y z^{2}\right)}{1+x\left(2 z+y z^{2}\right)}+\frac{1-y z}{1+y z}
$$

It is obvious, that the minimum of the real part is attained for $x=-1, y=1$, $z=|z|$, and the result is easily deduced.

REMARK 2. Since the extremum is attained by a function $f=z g, g \in P_{00}$, it is clear, that our result is best possible also in this subset.

3. Applications to $M$. At this stage we should make the following remark. Our results are obtained for subsets of $A_{0}$, but the members of all important classes in the theory of univalent functions in $U$ are of the form $z g(z), g \in A_{0}$. Since in our applications $z$ always occurs as a fixed number, it makes no difference in the proofs if we state our results for the subclass $A_{1} \subset A$, the members of which are normalized by $f(0)=0, f^{\prime}(0)=1$. According to this, we transform our classes $\hat{X}, \hat{Y}, \widehat{S t}, \hat{S}, \hat{M}, \hat{Z}$ into $X, Y, S t, S, M, Z$ by the general transformation $B=\{z f \mid f \in \hat{B}\}$.

In [9], [11] we proved the relation $M \subset S$ and that $M$ contains the class of normalized close-to-convex functions. In this special case Corollary 1 was already mentioned in [11]. Also Corollary 2 is of importance for $M$ : it is easily seen, 
that the extreme points of the closed convex hull of $X$ are represented by the functions

$$
\frac{z+1 / 2(y-x) z^{2}}{(1-x z)^{2}}, \quad|x|=|y|=1, x \neq y .
$$

Corollary 2, in connection with the Krein-Milman theorem, now proves that $f \in M$ has the representation

$$
f(z)=\int_{T} \frac{z+1 / 2(y-x) z^{2}}{(1-x z)^{2}} d \mu,
$$

where $\mu$ is a probability mass on $T=\{(x, y)|| x|=| y \mid=1\}$. This result, for the close-to-convex functions, was first deduced by L. Brickman, T. H. MacGregor and D. Wilken [1].

We now use our main theorem to obtain a result for $M$, which seems to be new even for close-to-convex functions.

THEOREM 4. Let $g \in M$. Then, for $n=1,2, \ldots$, we have

$$
\left|\frac{\pi_{n}(z, g)}{g(z)}-1\right| \leqslant(n+1+n|z|)|z|^{n} .
$$

Equality holds for the rotations of the Koebe function.

Proof. Putting $1 / 2(y / x-1)=-a /(a+1)$ it becomes clear, that it is enough to prove the theorem for the functions

$$
g(z)=\frac{1}{a+1}\left[\frac{a z}{1-x z}+\frac{z}{(1-x z)^{2}}\right], \quad \operatorname{Re} a \geqslant 0,|x| \leqslant 1 .
$$

In this case we have

$$
\begin{aligned}
\left|\frac{\pi_{n}(z, g)}{g(z)}-1\right| & =|x z|^{n}\left|\frac{a+n+1-x z(a+n)}{a+1-a x z}\right| \\
& \leqslant|z|^{n}\left(1+\frac{n}{|a+1 /(1-x z)|}\right) \\
& \leqslant|z|^{n}(1+n(1+|z|)) .
\end{aligned}
$$

The statement on the equality is easily checked.

The following inequality is known to be true for the members of several linear invariant subclasses of $S$, in particular for the close-to-convex functions. Since the elements of $X$ are close-to-convex the main theorem proves the same inequality for the members of $M$, which is not known to be linearly invariant.

THEOREM 5. Let $g \in M$. Then, for $n=2,3, \ldots$, we have the sharp inequality 


$$
\left|\frac{g^{(n)}(z)}{g^{\prime}(z)}\right| \leqslant n ! \frac{n+|z|}{(1-|z|)^{n+1}(1+|z|)} .
$$

4. Applications to $Z$. It is known [10], that $Z \subset S t$, and that the famous Bieberbach conjecture on univalent functions is equivalent to the statement $H \subset$ $Z$, where $H$ denotes the well-known subclass

$$
\left\{f=z+\sum_{k=2}^{\infty} a_{k} z^{k}\left|\sum_{k=2}^{\infty} k\right| a_{k} \mid \leqslant 1\right\}
$$

of $S t$. In this connection the question of the determination of the members of $Z$ is important and we restate that part of the main theorem for this special case:

THEOREM 6. Let $f \in A_{1} . f \in Z$ if and only if for every $\lambda \in \Lambda$ there exist $x, y \in \mathrm{C},|x|=|y|=1$, such that

$$
\lambda(f)=\lambda\left(\frac{z}{(1-x z)(1-y z)}\right) .
$$

The Marx conjecture [7], in its original version, states that the image of the circle $|z|<R, 0<R<1$, under the function $f^{\prime}(z)$, where $f$ is arbitrary in $S t$, is contained in the image of the same circle under the function $(1+z)(1-z)^{-3}$. This is known to be wrong with respect to the whole class St (J. A. Hummel [4]), but true for the functions $z /[(1-x z)(1-y z)],|x|=|y|=1$ (P. L. Duren and R. MacLaughlin [2]). Since $f^{\prime}(z)$, for fixed $z$, is a continuous linear functional on $A$, the main theorem proves:

THEOREM 7. The Marx conjecture is true for $Z$.

For $H$, however, the Marx conjecture is also true.

5. Applications of Corollary 3. In this part we prove two theorems which are typical for the use of Corollary 3. Both of them are extensions of known results.

THEOREM 8. Let $g(z) \in A_{1}, \operatorname{Re} e^{i \alpha} g(z) / z>0, z \in U$, for a suitable $\alpha \in \mathbf{R}$. Let $\beta \in \mathbf{R}$. Then the function $f_{\beta}=(1-\beta) g(z)+\beta z$ is univalent for

$$
|z|< \begin{cases}\left(\frac{2-2 \beta}{1-2 \beta}\right)^{1 / 2}-1, & \text { for } \beta \leqslant 1 / 10, \\ \left(\frac{\sqrt{\beta(1-\beta)}-\beta}{1-2 \beta}\right)^{1 / 2}, & \text { for } 1 / 10 \leqslant \beta \leqslant 1, \beta \neq 1 / 2, \\ 1 / \sqrt{2}, & \text { for } \beta=1 / 2, \\ 1-\left(\frac{2-2 \beta}{1-2 \beta}\right)^{1 / 2}, & \text { for } \beta \geqslant 1 .\end{cases}
$$

These bounds are sharp. 
Proof. We have $f_{\beta} / z \in P_{\beta}$. If we put, in Corollary $3, V=V_{\beta}, W=\hat{Y}$, $g=1 /(1-z)$, we may conclude, that the radius of $\hat{S}$-ness in $P_{\beta}$ equals the radius of $P_{\delta 0}$-ness in $\hat{Y}, \delta=(1-2 \beta) /(2-2 \beta)$. It is a well-known result, due to $\mathrm{A}$. Marx [7], that the possible values of the functional $f(z), f \in \widehat{S t} \supset \hat{Y}$ are already attained by the functions $(1-x z)^{-2},|x| \leqslant 1$. So it becomes clear, that the extreme values of $\operatorname{Re} f(z)$ in $\hat{Y}$ are taken by the functions $(1-x z)^{-2},|x|=1$, which belong to $\hat{Y}$. With this knowledge it is very easy to prove:

LEMma 3. For $f \in \hat{Y}$ we have the sharp estimate $\left({ }^{1}\right)$

$$
\frac{1}{(1-|z|)^{2}} \geqslant \operatorname{Re} f(z) \geqslant \begin{cases}\frac{1}{(1+|z|)^{2}}, & |z|<1 / 2, \\ \frac{1-2|z|^{2}}{2\left(1-|z|^{2}\right)^{2}}, & |z| \geqslant 1 / 2 .\end{cases}
$$

Using this lemma, there is no difficulty in evaluating the $P_{\delta 0}$-radius in $\hat{Y}$ for all real $\delta$, and a simple transformation $(\delta \rightarrow \beta)$ leads to the desired result.

RemarK 3. R. S. Gupta [3] proved, that for $g_{1} \in A_{1}, \operatorname{Re} g(z) / z>0, z \in U$, $0 \leqslant \beta \leqslant 1$, the radius of univalence and starlikeness of the functions $(1-\beta) g(z)$ $+\beta z$ is given by (2). It is possible, however, to evaluate the radius of starlikeness for the functions $f_{\beta}$ of Theorem 8 by Corollary $3\left(V=V_{\beta}, W=\hat{X}\right)$. In general this radius does not equal (2), according to the fact that the sharp bounds for $\operatorname{Re} f$ in $\hat{X}$ and $\hat{Y}$ are not equal.

It was shown by Y. Miki [8], that the partial sums of a function $f \in A_{1}$, which is univalent in $U$ and maps $U$ onto a convex domain, are convex and univalent for $|z|<1 / 4$. It is well known that these functions satisfy $\operatorname{Re} f(z) / z>1 / 2$, $z \in U$. We generalize Miki's result to all functions $f(z)=z g(z), g \in P_{1 / 2}$.

THEOREM 9. Let $f \in A_{1}, \operatorname{Re} e^{i \alpha}(f(z) / z-1 / 2)>0, z \in U$, for a suitable $\alpha \in \mathbf{R}$. Then all partial sums of $f(z)$ are univalent and convex for $|z|<1 / 4$.

Proof. In Corollary 3 we take $V=V_{1 / 2}, W=\hat{X}, g=\left(z \sigma_{n}(z)\right)^{\prime}$, where $\sigma_{n}(z)=\Sigma_{k=0}^{n} z^{k}$, and remark that for $h \in A_{0}$ we have $g * h=\left(z \pi_{n}(z, h)\right)^{\prime}$. It follows that the radius of $\widehat{S t}$-ness of $\left(z \pi_{n}(z, h)\right)^{\prime}$ for $h \in P_{1 / 2}$ equals the radius in which $\operatorname{Re}\left(z \pi_{n}(z, h)\right)^{\prime}>0$ for all $h \in \hat{X}$. We shall prove that this latter radius is $\geqslant 1 / 4$ for all $n \in \mathbf{N} \cup\{0\}$.

Assume that this has already been done and let $f$ be the function considered in the theorem. To prove our assertion, we have to show: $z\left(\pi_{n}(z, f)\right)^{\prime}$ is univalent and starlike for $|z|<1 / 4, n \in \mathrm{N}$. But $h(z)=f(z) / z \in P_{1 / 2}, \pi_{n}(z, f)=$

( $\left.{ }^{1}\right)$ This estimate is also valid in $\widehat{\text { St. }}$ 
$z \pi_{n-1}(z, h)$ and $\left(z \pi_{n-1}(z, h)\right)^{\prime}$ has radius of $\widehat{S t}$-ness not less than $1 / 4$ for $h \in P_{1 / 2}$. The result follows.

Since $\left(z \pi_{n}(z, h)\right)^{\prime}=\pi_{n}\left(z,(z h)^{\prime}\right), h \in A_{0}$, it remains to prove:

LEMma 4. For the functions $f(z)=(1-y z)(1-x z)^{-3},|x| \leqslant 1,|y| \leqslant 1$, we have $\operatorname{Re} \pi_{n}(z, f)>0$ for $|z|<1 / 4, n \in \mathrm{N} \cup\{0\}$.

The truth of the following lemma is easily verified.

LEMMA 5. Re $\sigma_{n}(z)>1 / 2$ for $|z|<r_{n}$, where $r_{n}$ is the positive root of $r^{n+1}$ $=(1-r) / 2$. This bound is sharp for $n$ odd.

Proof of Lemma 4. Clearly $\operatorname{Re} f(z)>0$ for $|z|<\sin (\pi / 8)=0.3826 \cdots$. For $n \geqslant 4$ we have $\operatorname{Re} \sigma_{n}>1 / 2$ for $|z|<0.685$, so we can conclude $\operatorname{Re} \pi_{n}(z, f)$ $=\operatorname{Re}\left(\sigma_{n} * f\right)(z)>0$ for $|z|<0.3826 \cdot 0.685=0.26 \ldots$ and it remains to prove the cases $n=0,1,2,3$. Obviously the extrema will be attained for $|x|=$ $|y|=1$, and without loss of generality we may assume $x=1$. It follows that

$$
\pi_{n}(z, f)=\sum_{k=1}^{n+1} k \frac{k+1-y(k-1)}{2} z^{k-1},
$$

and the condition $\operatorname{Re} \pi_{n}(z, f) \geqslant 0$ is equivalent to

$$
\left|\sum_{k=2}^{n+1} \frac{k(k-1)}{2} z^{k-1}\right| \leqslant \operatorname{Re} \sum_{k=1}^{n+1} \frac{k(k+1)}{2} z^{k-1} .
$$

The case $n=0$ is trivial and, for $n=1,(3)$ is true and sharp for $|z| \leqslant 1 / 4$. Now let $n=2$. Then, for $|z| \leqslant 1 / 4$, we have

$$
\left|z+3 z^{2}\right| \leqslant 7 / 16, \quad \operatorname{Re}\left(1+3 z+6 z^{2}\right) \geqslant 7 / 16,
$$

and (3) follows.

The case $n=3$ requires some more work. It is enough to prove (3) for $|z|$ $=1 / 4$. If we put $z=e^{i \varphi} / 4, \cos \varphi=x$, we obtain

$$
\operatorname{Re}\left(1+3 z+6 z^{2}+10 z^{3}\right)=\frac{5}{8}+\frac{9}{32} x+\frac{3}{4} x^{2}+\frac{5}{8} x^{3}=F(x) .
$$

For $x \geqslant 0$ it follows $F(x) \geqslant F(0)=5 / 8$, and for $-1 \leqslant x<0: F(x) \geqslant F(-1)$ $=15 / 32$. On the other hand, for $x \geqslant 0$, we have $\left|z+3 z^{2}+6 z^{3}\right| \leqslant 17 / 32<$ $5 / 8$, and for $x<0$

$$
\begin{aligned}
\left|z+3 z^{2}+6 z^{3}\right| & =1 / 4 \sqrt{1+9|z|^{2}+36|z|^{4}+12 \operatorname{Re}\left(z^{2}\right)+\left(6+18|z|^{2}\right) \operatorname{Re} z} \\
& \leqslant 1 / 4 \sqrt{1+21|z|^{2}+36|z|^{4}}<13 / 32 .
\end{aligned}
$$

The proof is now completed. 


\section{REFERENCES}

1. L. Brickman, T. H. MacGregor and D. R. Wilken, Convex hulls of some classical families of univalent functions, Trans. Amer. Math. Soc. 156 (1971), 91-107. MR 43 \#494.

2. P. L. Duren and R. McLaughlin, Two-slit mappings and the Marx conjecture, Michigan Math. J. 19 (1972), 267-273. MR 46 \#3762.

3. R. S. Gupta, Radius of convexity of a class of univalent functions, J. Indian Math. Soc. 36 (1972), 291-296.

4. J. A. Hummel, The Marx conjecture for starlike functions, Michigan Math. J. 19 (1972), 257-266. MR 46 \#3761.

5. J. L. Kelley and I. Namioka, Linear topological spaces, University Ser. in Higher Math., Van Nostrand, Princeton, N. J., 1963. MR 29 \#3851.

6. T. H. MacGregor, Functions whose derivative has a positive real part, Trans. Amer. Math. Soc. 104 (1962), 532-537. MR 25 \#4090.

7. A. Marx, Untersuchungen über schlichte Abbildungen, Math. Ann. 107 (1932), 40-67.

8. Y. Miki, A note on close-to-convex functions, J. Math. Soc. Japan 8(1965), 256-268.

9. S. Ruscheweyh, Über die Faltung schlichter Funktionen, Math. Z. 128 (1972), 85-92. MR 47 \#7017.

10. S. Ruscheweyh and K.-J. Wirths, Über die Faltung schlichter Funktionen. II, Math. Z. 131 (1973), 11-23. MR 47 \#8836.

11. S. Ruscheweyh and T. Sheil-Small, Hadamard products of schlicht functions and the Pólya-Schoenberg conjecture, Comment. Math. Helv. 48 (1973), 119-135.

12. O. Toeplitz, Die linearen vollkommenen Räume der Funktionentheorie, Comment. Math. Helv. 23 (1949), 222-242. MR 11, 372.

DEPARTMENT OF MATHEMATICS, UNIVERSITY OF DORTMUND, DORTMUND, FEDERAL REPUBLIC OF GERMANY 